\title{
Bayesian approach to quantify parameter uncertainty and impacts on predictive flow and mass transport in heterogeneous aquifer
}

\author{
J. Liang $\cdot$ G. M. Zeng $\cdot$ S. Shen $\cdot$ S. L. Guo $\cdot$ \\ X. D. Li $\cdot$ Y. Tan $\cdot$ Z. W. Li $\cdot$ J. B. Li
}

Received: 10 April 2012/Revised: 16 June 2013/Accepted: 24 November 2013/Published online: 18 December 2013

(C) Islamic Azad University (IAU) 2013

\begin{abstract}
Groundwater flow and mass transport predictions are subjected to uncertainty due to heterogeneity of hydraulic conductivity, whose variability in space is considerably higher than that of other hydraulic properties relevant to groundwater flow. To characterize the distribution of hydraulic conductivity, random space function (RSF) is often used. The Bayesian approach was applied to quantitatively study the effect of parameter uncertainty in RSF on a hypothetical two-dimensional uniform groundwater flow and mass transport. Specifically, the parameter uncertainty transmitted to macrodispersion in mass transport model was also inferred. The results showed that the posterior probability distributions of parameters were updated after Bayesian inference. The numerical experiments indicated that the overall predictive uncertainty was increased with simulating time along the flow direction. As to the relative contribution of the two types of uncertainty, it indicated that parametric uncertainty was a little more
\end{abstract}

J. Liang $(\bowtie) \cdot$ G. M. Zeng · S. Shen · S. L. Guo ·

X. D. Li · Y. Tan · Z. W. Li · J. B. Li

College of Environmental Science and Engineering, Hunan

University, Changsha 410082, People's Republic of China

e-mail: liangjie82@163.com

J. Liang - G. M. Zeng - S. Shen · X. D. Li · Y. Tan ·

Z. W. Li · J. B. Li

Key Laboratory of Environmental Biology and Pollution Control (Hunan University), Ministry of Education, Changsha 410082,

People's Republic of China

S. L. Guo

State Key Laboratory of Water Resources and Hydropower Engineering Science, Wuhan 430072,

People's Republic of China important than stochastic uncertainty for the predictive uncertainty of hydraulic head. When the uncertainty of hydraulic head as well as macrodispersion was transported to mass transport model, a much bigger contribution of stochastic uncertainty was observed. Therefore, parametric uncertainty should not be neglected during the process of subsurface simulation.

Keywords Groundwater - Heterogeneous - Bayesian approach · Uncertainty · Hydraulic conductivity

\section{Introduction}

A major difficulty in accurately describing and simulating subsurface flow and mass transport arises from the uncertainty of subsurface system. The uncertainty stems from a number of factors including parametric uncertainty, spatial variability, concept uncertainty and boundary uncertainty (Rojas et al. 2010). Among them, parametric uncertainty caused by heterogeneity of geological properties has aroused widely attention. Hydraulic conductivity, which reflects the characteristics of porous media, is considered to be the most important uncertain input parameter of groundwater flow models (Zeng et al. 2009). Its variability in space is considerably higher than that of other hydraulic properties relevant to groundwater flow, and it can vary by orders of magnitude over a few meters (Feyen et al. 2003).

Hydraulic conductivity varies in space in a nondeterministic way, generally following some kind of structural pattern. To characterize the spatial variability of the hydraulic conductivity, random space function (RSF) is often adopted (Ezzedine and Rubin 1996; Feyen et al. 2003; Franssen et al. 2003; Kerrou et al. 2008; Hassan et al. 2009; Liang et al. 2009, 2010). However, scarcity of 
measurements leads to generating spatial structure pattern of hydraulic conductivity at a few sampling location, which arouse uncertainty of spatial hydraulic conductivity distribution. In practice, the parameters are inferred conditional to measurements in a process known as "parameter estimation". In fact, the practice significance of parameter estimation is more apparent as models improve in their description of physical processes. The development of accurate parameter estimation methods holds the promise of allowing the full utilization of measurements, which are collected at high cost, in achieving the best possible site characterization. Many articles have been published on this subject (Yeh 1986; Sun 1994), and many of the currently available methods have been developed, including classical deterministic approach related to generalizations of the least squares or maximum-likelihood approach (Yeh and Yoon 1981) and Bayesian approach. The latter can describe natural uncertainties in the model parameters from limited and imperfect data. The inferred results expressed in terms of probability distribution are known as the posterior distribution, which can be used to summarize parameter uncertainty and quantify its effects on model prediction. Parameter uncertainty is quantified by a prior probability distribution, which represents historical or expert information before any new data are collected, and a likelihood function, which characterizes the proximity of simulated and observed data. Feyen et al. (2003) were the first to pay attention to the uncertainty of the parameters in RSF and apply Bayesian approach to infer them. They found that the predictions of the Bayesian approach was more conservative compared with classical approach. The same results were also obtained by Franssen et al. (2003) under the framework of Bayesian approach.

The uncertainty of hydraulic conductivity can cause the changes of other input parameters. The macrodispersion, one of the important parameters of groundwater mass transport model, can be viewed as a parameter reflecting the spatial variability of hydraulic conductivity. Experimental and theoretical results have suggested that macrodispersion of solute is essentially produced by the spatial variation of the fluid velocity resulting from the heterogeneity of hydraulic conductivity (Fu and Gómez-Hernández 2009). Therefore, the uncertainty of macrodispersion caused by uncertainty of hydraulic conductivity should be included in the analysis.

The Bayesian approach provides a rigorous framework to represent the uncertainty lies in the parameter in natural. The parameter is then stochastically chosen from its inferred posterior probability distribution using Monte Carlo (MC) sampling technique and is used to generate spatial distribution of hydraulic conductivity, which is the input of groundwater flow model. The uncertainty of parameters in RSF and macrodispersion is then transported to groundwater flow and mass transport model. During the process related above, two types of uncertainty, including parametric uncertainty and stochastic uncertainty, lie in. This paper demonstrates the use of Bayesian approach to estimate posterior probability distribution of parameters in RSF and macrodispersion. With each drawn sample, the groundwater flow and mass transport model must be run to produce the output that is compared with the reference data to quantificationally characterize the uncertainty. Specially, we focus on addressing the contribution of stochastic uncertainty and parametric uncertainty on the predictive hydraulic head and mass concentration. This paper is organized as follows: Section "Materials and methods" presents a brief background about the Bayesian approach and the relationship between hydraulic conductivity and macrodispersion. In Sect. "Results and discussion", a hypothetical steady two-dimensional flow and transport field is given to test the parameter uncertainty of predictive mass transport. Results and a summary of the key aspects of the achieved uncertainty reduction in model input as well as output are also presented. The main conclusions drawn from the analysis are presented in Sect. "Conclusion."

\section{Materials and methods}

Spatial stochastic hydraulic conductivity field

Hydraulic conductivity $(K)$ is found to be $\log$-normally or $\ln$-normally distributed in a heterogeneous aquifer (Sudicky 1986). Let $K(x)$ denote the stochastic hydraulic conductivity field, and its $\ln$ form is denoted as $Y(x)=\ln K(x)$, where $x$ is the unmeasured spatial location. Gaussian stationary RSF, with a constant average value of $\ln K(x)$ and an isotropic exponential two-point covariance function, is adopted to quantitative describe the spatial continuity of $Y(x)$ :

$$
\begin{aligned}
& \overline{Y(x)}=\mu \\
& V(h)=[Y(x+h)-\overline{Y(x+h)}][Y(x)-\overline{Y(x)}]=\sigma^{2} \rho(h) \\
& V(h)=\sigma^{2} \exp (|h| / \varphi)
\end{aligned}
$$

where $\mu$ is the expected value or average value of $\ln K(x)$, $V(h)$ is the two-point covariance function of the process with lag separation vector $h$, the variance $\sigma^{2}$ and the correlation function $\varphi$ is the integral scale of the spatial stochastic process. To generate hydraulic conductivity as input of groundwater flow model, $\mu, \sigma^{2}$ and $\varphi$ should be determined from limited measured data. Here, we denote parameters as $\theta=\left(\mu, \sigma^{2}, \varphi\right)$. The process of parameters estimation introduces uncertainty from inability to exactly 


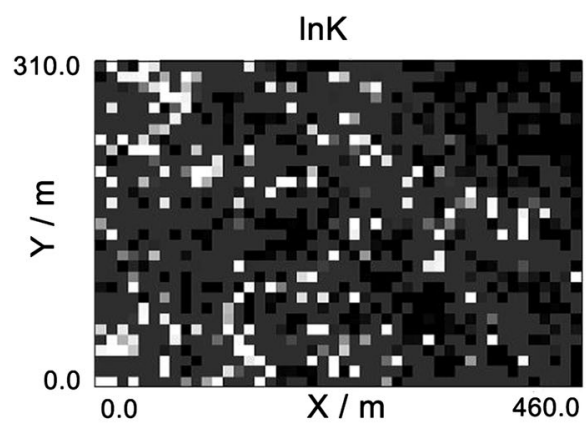

(a)

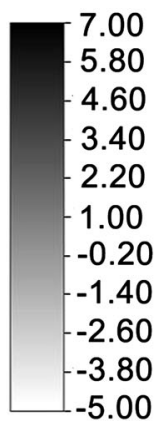

5.00

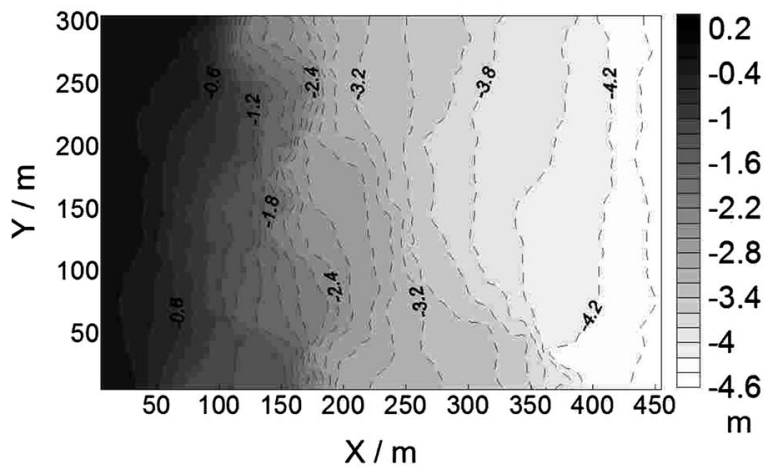

(c)

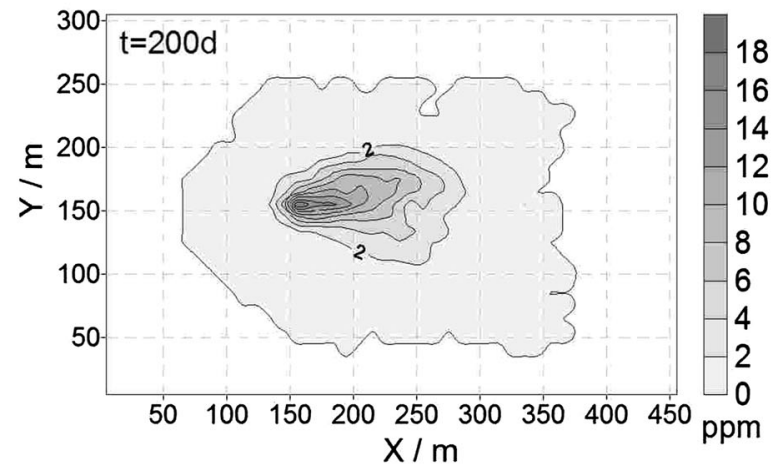

(e)

Fig. 1 Hypothetical distribution of hydraulic conductivity, hydraulic head and mass concentration: a hypothetical hydraulic conductivity field, b locations of sampled hydraulic conductivity, c reference distribution of hydraulic head, $\mathbf{d}$ reference distribution of mass

estimate hydraulic conductivity at unmeasured locations, which should be considered in prediction.

\section{Macrodispersion}

Macrodispersion is an important parameter of groundwater mass transport model. It is defined as the change rate of the second-order moment of a mass plume. Studies on the effects of hydraulic conductivity on macrodispersion of solutes have shown the macrodispersion coefficients that

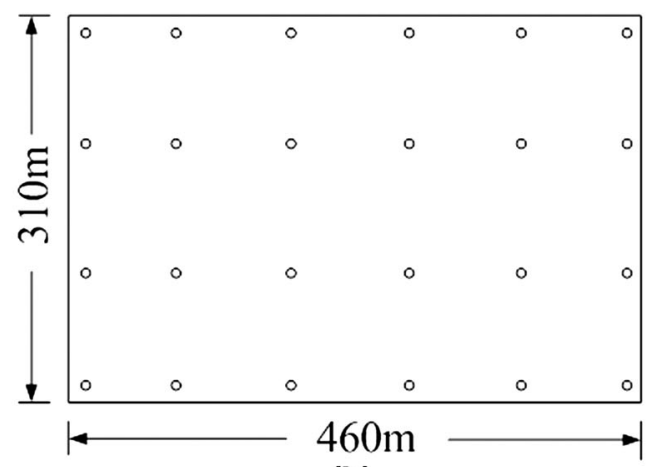

(b)

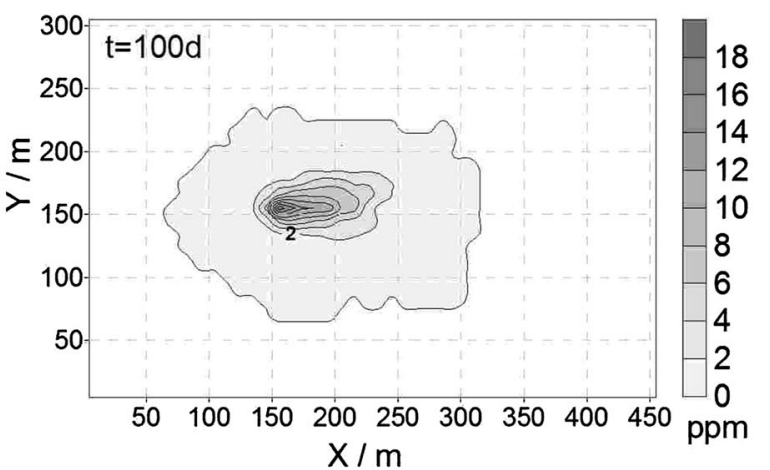

(d)

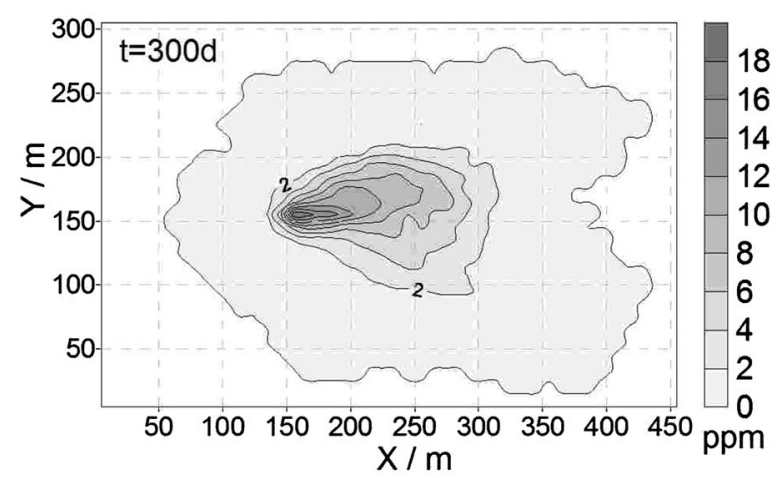

(f)

concentration for $t=100$ days, e reference distribution of mass concentration for $t=200$ days, and $\mathbf{f}$ reference distribution of mass concentration

can be calculated from the statistical of hydraulic conductivity. We compute the macrodispersion coefficients according to Eq. 4

$A_{L}=\frac{\sigma_{\ln K}^{2} \lambda_{L}}{\gamma^{2}}$

where $A_{L}$ is the apparent longitudinal macrodispersion along the groundwater flow direction, $\sigma_{\ln K}^{2}$ is the variance of $\ln K, \lambda_{L}$ is the relevant scale of $\ln K$, and $\gamma$ is experience flow factor. 
Table 1 Prior distribution of parameters

\begin{tabular}{lll}
\hline Parameter & Prior probability distribution & Hypothetical value \\
\hline$\mu$ & Uniform [0, 5] & 4.1052 \\
$\sigma^{2}$ & Uniform [0, 2] & 0.5 \\
$\varphi$ & Uniform [0, 300] & 200 \\
\hline
\end{tabular}

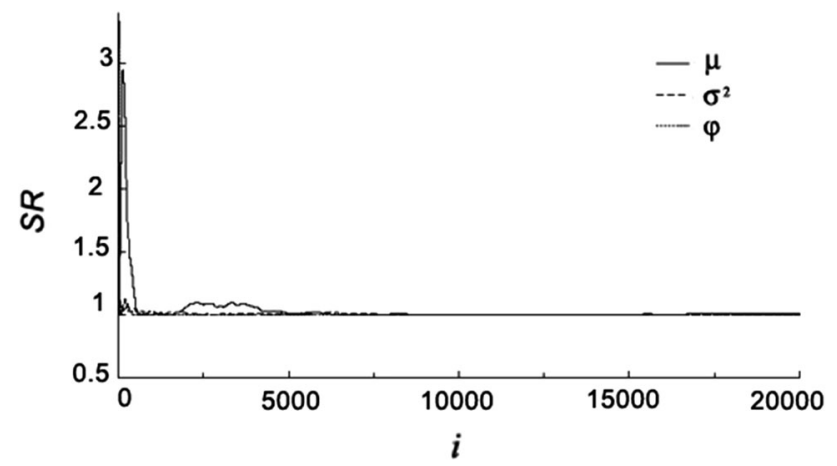

Fig. 2 Evolution of the SR score for parameter $\theta=\left(\mu, \sigma^{2}, \varphi\right)$ using $\mathrm{MH}$ algorithm

Bayesian approach to estimate parameter

Bayesian approach can deal with uncertainty that stems from the imperfect knowledge of parameters. In Bayesian
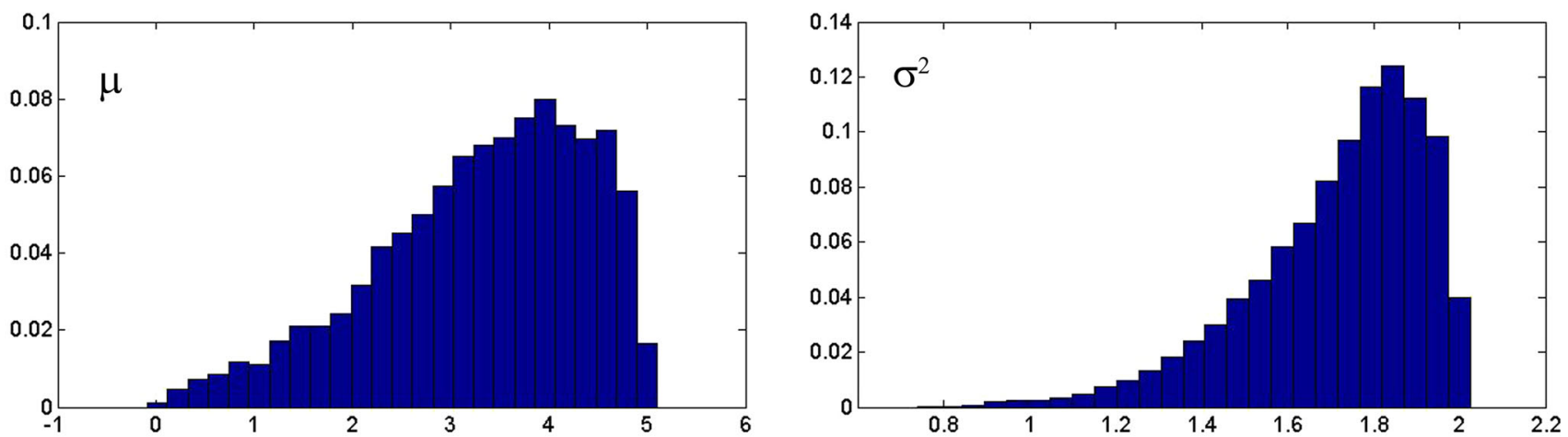

approach, the parameter $\theta$ is characterized by posterior probability distribution. It is obtained by combining the prior information about the parameters before the measurements are introduced with the likelihood information about the proximity of simulated and observed data according to Bayes' rules. The prior information can be obtained from borehole description, expert judgments, previous studies, etc., and the likelihood information can be obtained from the likelihood function. Assume that the $\ln$ form of observed hydraulic conductivity is $y=\left(y_{1}\right.$, $\left.y_{2}, \ldots, y_{n}\right)^{T}$, on the basis of Bayesian inference, the resulting predictive posterior distribution of the $\ln K$ is obtained

$p(Y(x) \mid y)=\int p(Y(x) \mid y, \theta) p(\theta \mid y) d \theta$

where $p(\theta \mid y)$ is the prior probability distribution, $p(Y(x) \mid y$, $\theta)$ is the likelihood function and $p(Y(x) \mid y)$ is the posterior probability distribution.

The realization of $\ln \mathrm{K}$ is MC sampled posterior from its probability distribution and transformed to $K$, which is used as inputs of groundwater flow model. The predictive hydraulic head is given by:

$p(H(x, t) \mid y)=\int f[p(Y(x) \mid y, \theta) p(\theta \mid y), t]$

The posterior probability distribution of $\theta$ was applied to calculate $A_{L}$, which is used as one of input parameters

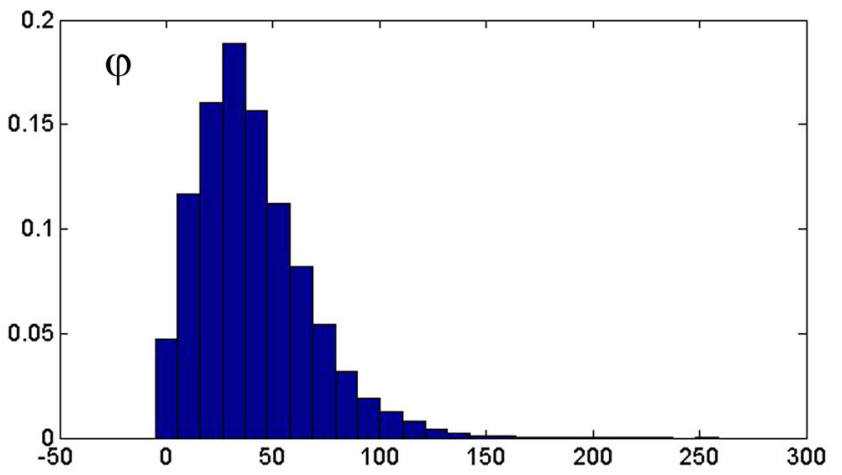

Fig. 3 Marginal posterior probability distributions for parameter $\theta=\left(\mu, \sigma^{2}, \varphi\right)$ 
Table 2 Statistic of posterior distribution for parameters

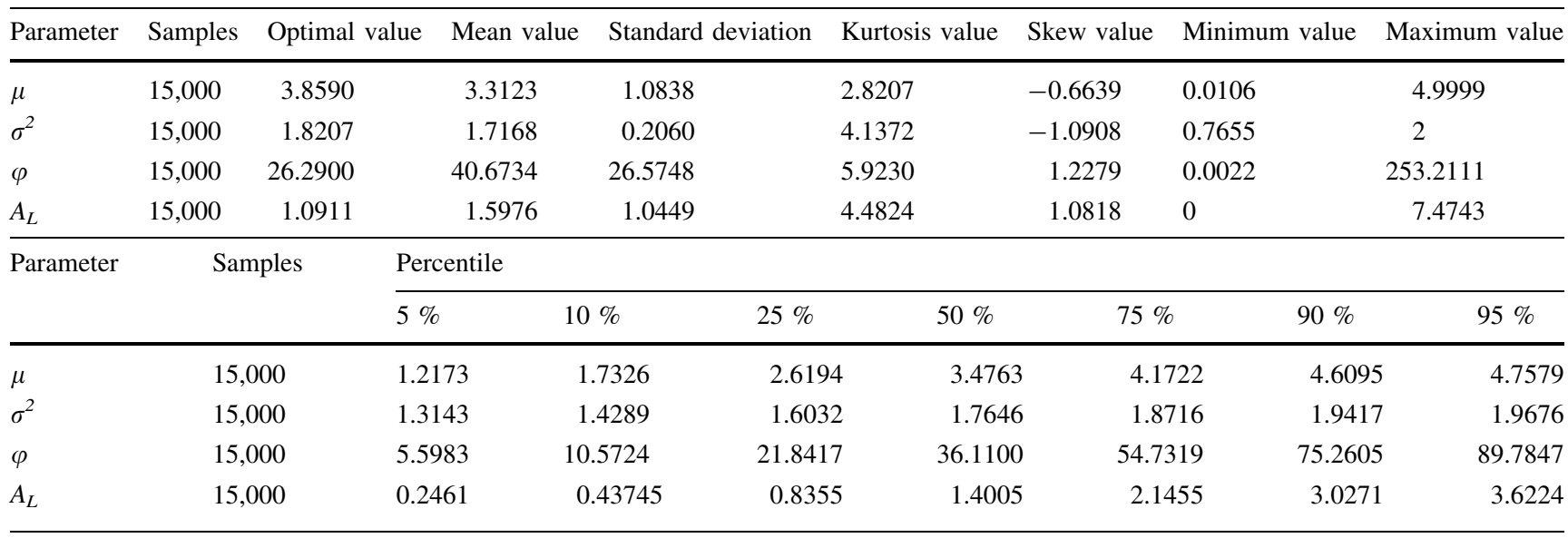

Table 3 Assessment of hydraulic head uncertainty

\begin{tabular}{llll}
\hline Uncertainty index & $\mathrm{I}_{1}$ & $\mathrm{I}_{2}$ & $\mathrm{I}_{3}$ \\
\hline$U_{1}$ & 0.0328 & 0.3634 & 0.2022 \\
$U_{2}$ & 0.2667 & 0.3993 & 0.2946 \\
$U$ & 0.2981 & 0.4588 & 0.3887 \\
\hline
\end{tabular}

Table 4 Assessment of mass transport uncertainty

\begin{tabular}{lllll}
\hline Uncertainty index & Time d & $I_{1}$ & $I_{2}$ & $I_{3}$ \\
\hline$U_{1}$ & 100 & 1.5568 & 0.2377 & 2.0291 \\
& 200 & 2.7255 & 0.4239 & 3.3235 \\
& 300 & 3.8254 & 0.6820 & 4.7610 \\
$U_{2}$ & 100 & 1.5190 & 0.1991 & 1.7438 \\
& 200 & 2.5556 & 0.3921 & 3.1026 \\
& 300 & 3.0350 & 0.6197 & 4.5693 \\
$U$ & 100 & 1.6825 & 0.2861 & 2.2633 \\
& 200 & 3.4180 & 0.4683 & 4.1374 \\
& 300 & 5.1101 & 0.7104 & 6.1422 \\
\hline
\end{tabular}

of mass transport model. After numerical simulation, the predictive distribution of mass transport is given by:

$p(C(x, t) \mid y)=\int f\left[p(Y(x) \mid y, \theta) p(\theta \mid y), A_{L}, t\right]$

The Bayesian predictive hydraulic heads and mass distribution can be seen as the transformation of the predictive $\ln K$ and $A_{\mathrm{L}}$, with the function given by the groundwater flow and mass transport equation. Therefore, through Bayesian inference, the predictive hydraulic head and mass distribution are conditional on the measurements only, and thus, the uncertainty about parameters and stochastic simulation is incorporated in the predictions.

Uncertainty assessment

To assess predictive uncertainty of mass transport in groundwater, three statistics are defined. $I_{1}$ measures the precision of the realizations since it evaluates the ensemble variance over all the cells. $I_{2}$ and $I_{3}$ take the advantage of the knowledge of the reference field to measure the average bias and a combination of bias and precision, respectively. The statistics are defined below: 

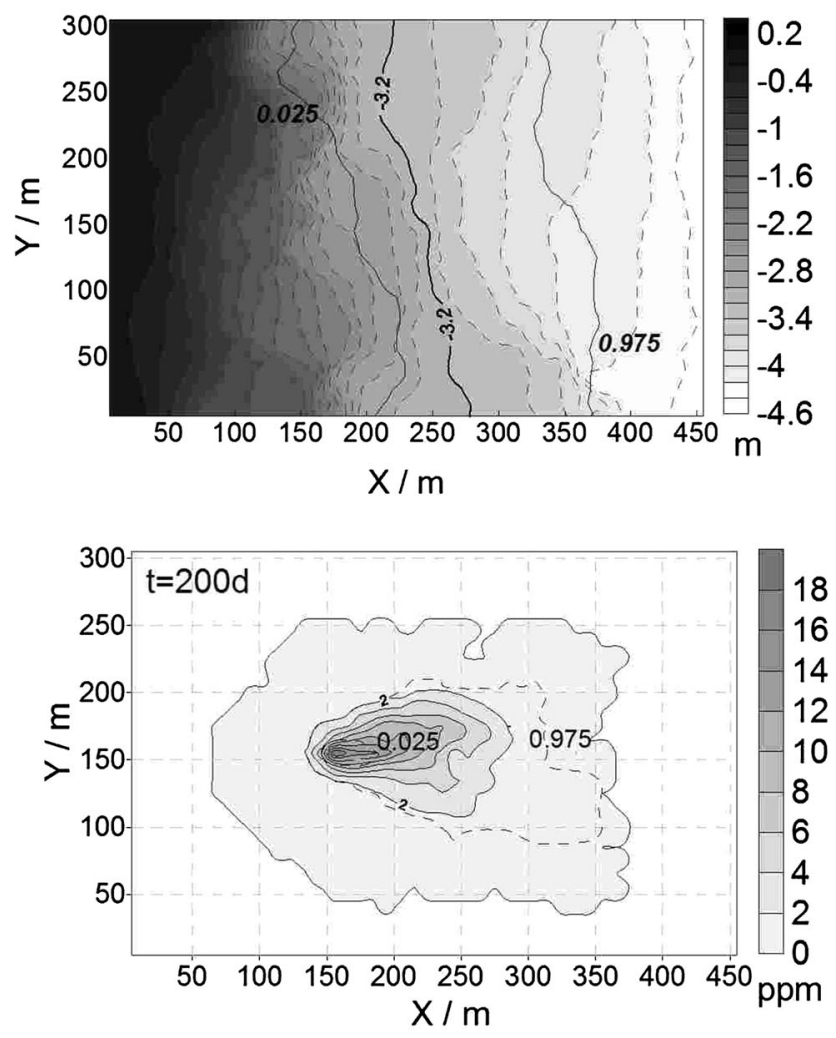
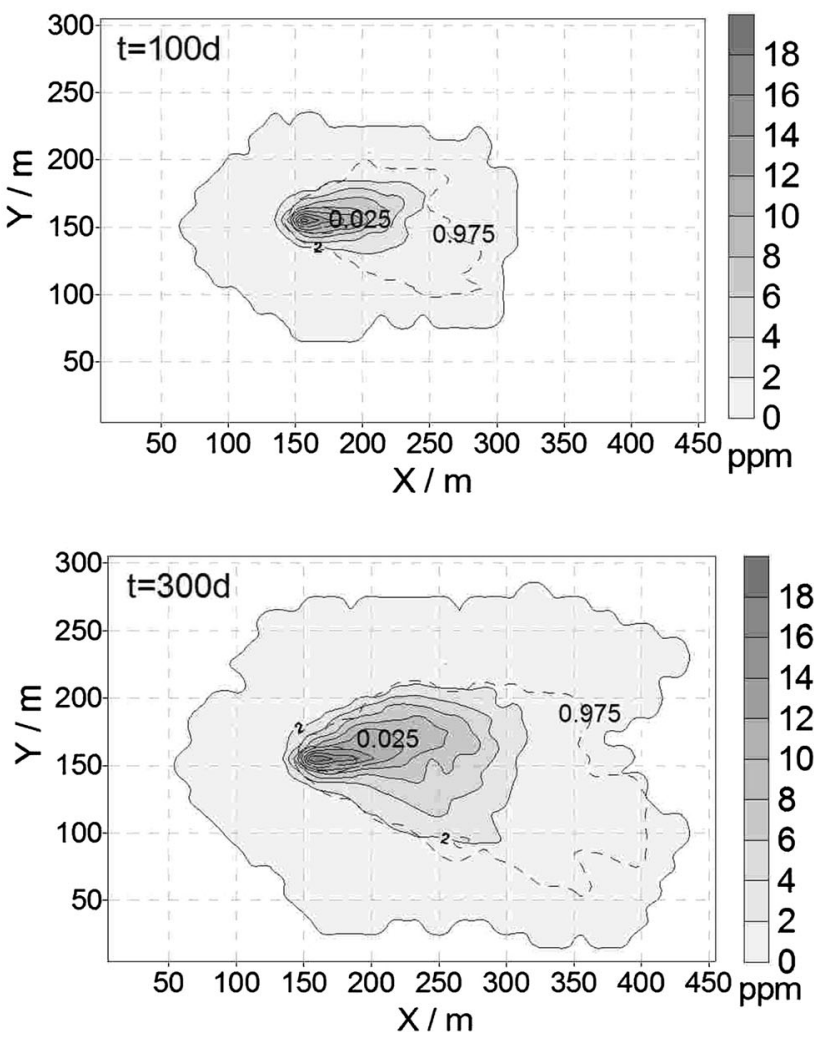

Fig. 4 Ensemble average hydraulic head and mass concentration distributions for $t=100$ days, $t=200$ days and $t=300$ days: stochastic uncertainty

$I_{1}=\frac{1}{n} \sum_{i=1}^{n} \frac{1}{n_{r}} \sum_{r=1}^{n_{r}}\left(x_{i, r}-\overline{x_{i}}\right)^{2}$

$I_{2}=\frac{1}{n} \sum_{i=1}^{n} \frac{1}{n_{r}} \sum_{r=1}^{n_{r}}\left|x_{i, r}-x_{i, r e f}\right|$

$I_{3}=\frac{1}{n} \sum_{i=1}^{n} \frac{1}{n_{r}} \sum_{r=1}^{n_{r}}\left(x_{i, r}-x_{i, r e f}\right)^{2}$

where $n$ is the number of cells, $n_{r}$ is the number of realizations, $x_{i, r}$ is the simulated hydraulic head or mass concentration value at cell $i$ and realization $r, \overline{x_{i, r}}$ is the ensemble average hydraulic head or mass concentration value over all realizations at cell $i$, and $x_{i, r e f}$ is reference hydraulic head or mass concentration value at cell $i$.

\section{Results and discussion}

Study domain

A hypothetical steady two-dimensional flow and transport field in the groundwater constructed by Wilson and Miller
(1978) is adopted to reflect the subsurface flow and solute transport under the generated hydraulic conductivity field. The study domain is depicted in plan view in Fig. 1 with a grayscale overlay of the distribution of $\ln K(x)$ generated by the unconditional sequential Gaussian simulation (sgsim) algorithm. The study domain is $460 \times 310 \mathrm{~m}^{2}$. A point source releases a small amount of fluid into the aquifer at $1 \mathrm{~m}^{3} /$ day at location of $(x=160, y=160)$. The injected fluid carries a nonreactive solute at a concentration of 1,000 ppm. The contaminant migrates by advection and dispersion at a Darcy velocity of $0.33 \mathrm{~m} /$ day. The porosity of the porous medium is 0.3 .

The flow model is surrounded by constant-head boundaries on the east and west borders and no-flow boundaries on the north and south borders of the study area. The aquifer is initially pristine with concentrations everywhere equal to zero. The only source of contaminant is the injection, so flow through the inlet has zero concentration. The steady groundwater flow is simulated using MODFLOW-96, and the mass transport is simulated using MT3DMS through the third-order total-variation-diminishing solution scheme. 

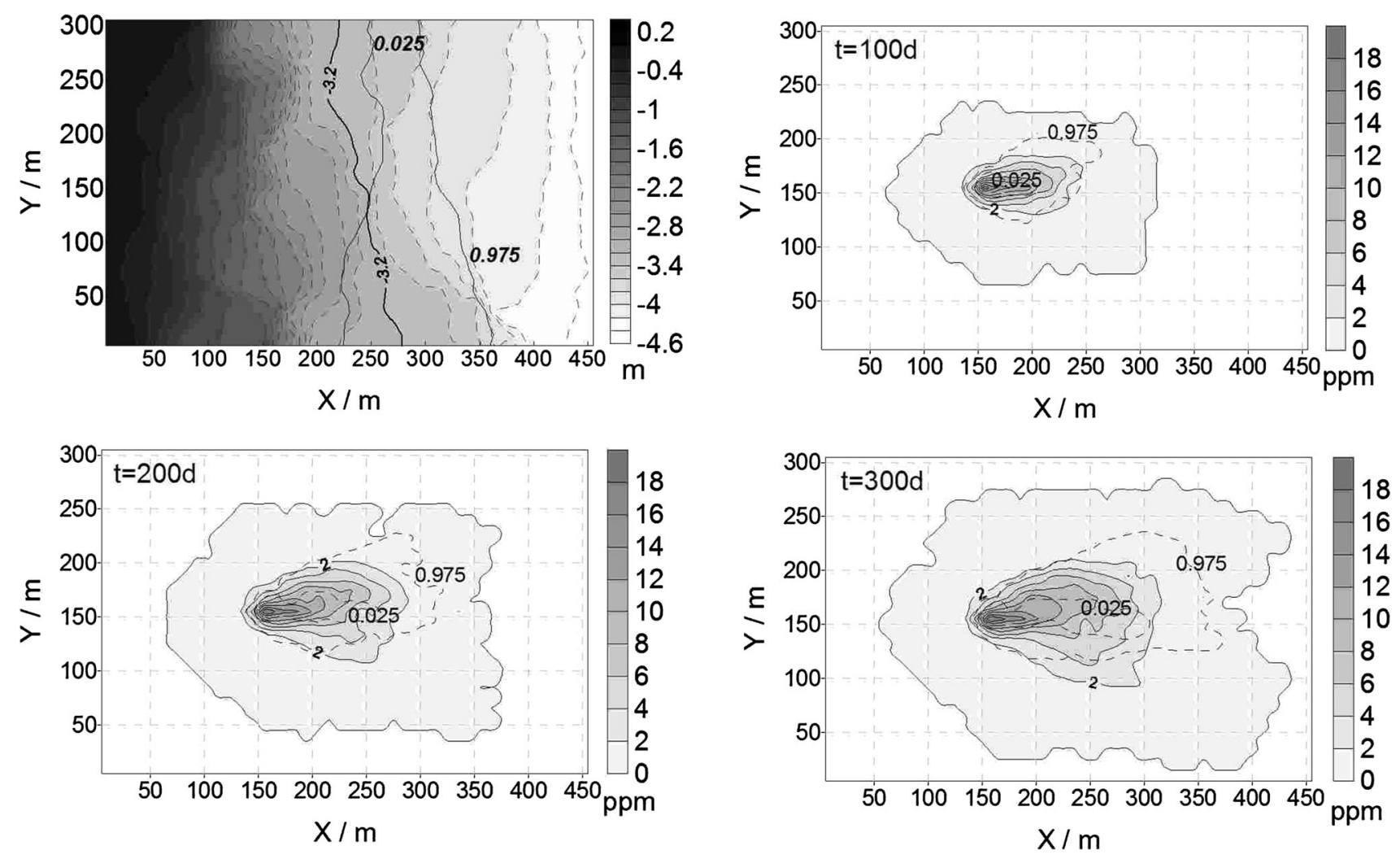

Fig. 5 Ensemble average hydraulic head and mass concentration distributions for $t=100$ days, $t=200$ days and $t=300$ days: parametric uncertainty

The sets of $\ln K(x)$ measurements are taken regularly from the study domain of the hypothetical field in Fig. 1. The study domain is discretized into 46 columns along $y$ axis and 31 rows along $x$-axis. The number and the location of the measurement taken from the study domain are the same to remove the effect of measurements. First, the measurements are used to update prior information to generate the posterior probability distribution of parameter $\theta$. Next, the conditional $\ln K(x)$ field is yielded from $\theta$ using conditional sgsim algorithm according to the spatial scale of $\ln K(x)$. Then, $\ln K(x)$ field is transformed to $K$ field and calculated $A_{L}$. Lastly, $K$ and $A_{L}$ are used as inputs of groundwater flow and transport model, respectively.

Predictive posterior of parameter

In the Bayesian approach to estimate parameter $\theta$, we assumed that no information about $\theta$ was present. Therefore, uniform distribution is specified as prior dis- tribution of $\theta$. However, in real applications, prior information about the parameters may be available and more informative priors can be specified. The prior probability distribution and hypothetical value of $\theta$ can be seen in Table 1. Markov Chain Monte Carlo (MCMC) approach and specifically the Metropolis-Hastings (MH) algorithm are applied to update the prior probability distribution. The MCMC MH algorithm is run with $q=5$ parallel sequences with each having a population size of $s=20,000$. The likelihood of parameter $\theta$ given the measurements $y$ is computed using the function described by Box and Tiao (1992), and the convergence of the algorithm is assessed using the Scale Reduction (SR) score $(\sqrt{S R})$ defined by Gelman and Rubin (1992). If $\sqrt{S R}$ is $<1.0$, the Markov chain is considered to be converged; otherwise, more runs are needed. In Fig. 2, the calculated values of $\sqrt{S R}$ are plotted against the number of MCMC iterations. The line plots indicated that for parameter $\sigma^{2}$ and $\varphi$, the parallel sequences converged to the target distribution rapidly and, for 
parameter $\mu$, the parallel sequences converged to the target distribution after approximately 6,000 iterations.

Using Bayesian approach, the posterior probability distribution for the parameter $\theta$ is obtained. The posterior distribution of $\theta$ is obtained by statistic generated parameter values in $\mathrm{MC}$ chains, removing the values of the initial 6,000 times oscillation. Figure 3 presents the marginal posterior probability distributions for parameter $\theta$. The distributions did not resemble a standard probability distribution. The summary statistics of the marginal posterior parameter distributions are given in Table 2 . The parameter $\theta$ is transported to $A_{L}$, and the calculated $A_{L}$ is also listed in Table 2. Among them, the optimal value of parameter is decided by dividing the parameter space into 25 groups and taking the median of a group with highest probability. Percentiles help to determine the confidence interval of parameter and thus reflects the uncertainty of parameter, for example, $90 \%$ confidence interval is (5th percentile value, 95th percentile value), $80 \%$ confidence interval is (10th percentile value, 90th percentile value), and the rest may be deduced by analogy. The histograms and the statistics showed that the posterior probability distributions of parameter $\theta$, which did not resemble uniform distribution, were updated after Bayesian inference.
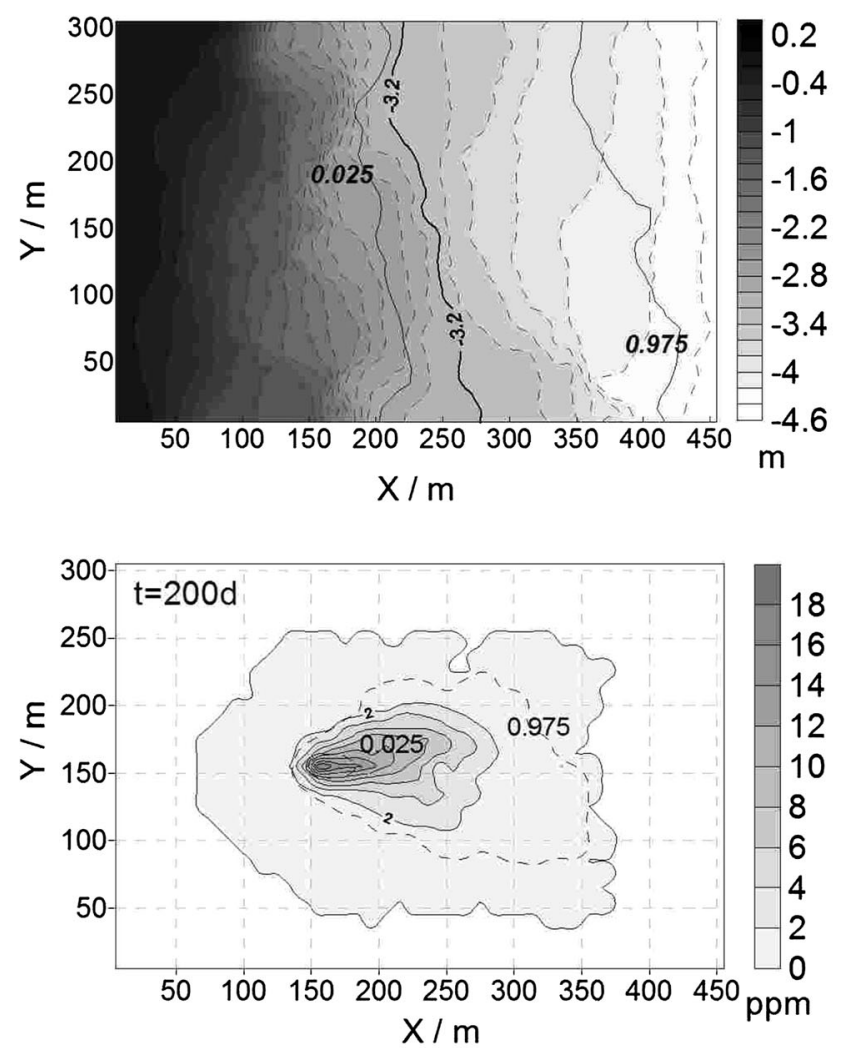

Uncertainty assessment of hydraulic head and mass transport

Two types uncertainty for predictive hydraulic head and mass transport are assessed in the paper, including stochastic uncertainty $\left(U_{1}\right)$ and parametric uncertainty $\left(U_{2}\right)$. The overall predictive uncertainty $(U)$, which is contributed by the two types of uncertainty, is also evaluated. To assess uncertainty of $U_{1}, U_{2}$ and $U, \mathrm{MC}$ sampling is used to generate 100 sets of parameter $\theta$ from the posterior probability distribution. Each set is used to yield 100 alternative images of the hydraulic conductivity field by sgsim, which is used as inputs of the groundwater flow and to update $A_{L}$. The corresponding uncertainty assessment index $I_{1}, I_{2}$ and $I_{3}$ are calculated and listed in Tables 3 and 4. Statistical analysis of the ensemble of predictive hydraulic head and predictive mass transport for $t=100$ days, $t=200$ days and $t=300$ days are given in Figs. 4, 5 and 6. The calculated uncertainty assessment index $I_{1}, I_{2}$ and $I_{3}$ in Tables 3 and 4 showed that stochastic uncertainty $\left(U_{1}\right)$ and parametric uncertainty $\left(U_{2}\right)$ were contributed to the overall predictive uncertainty $(U)$ for hydraulic head and mass concentration simultaneously, which should not be neglected during the process of simulation. For all time
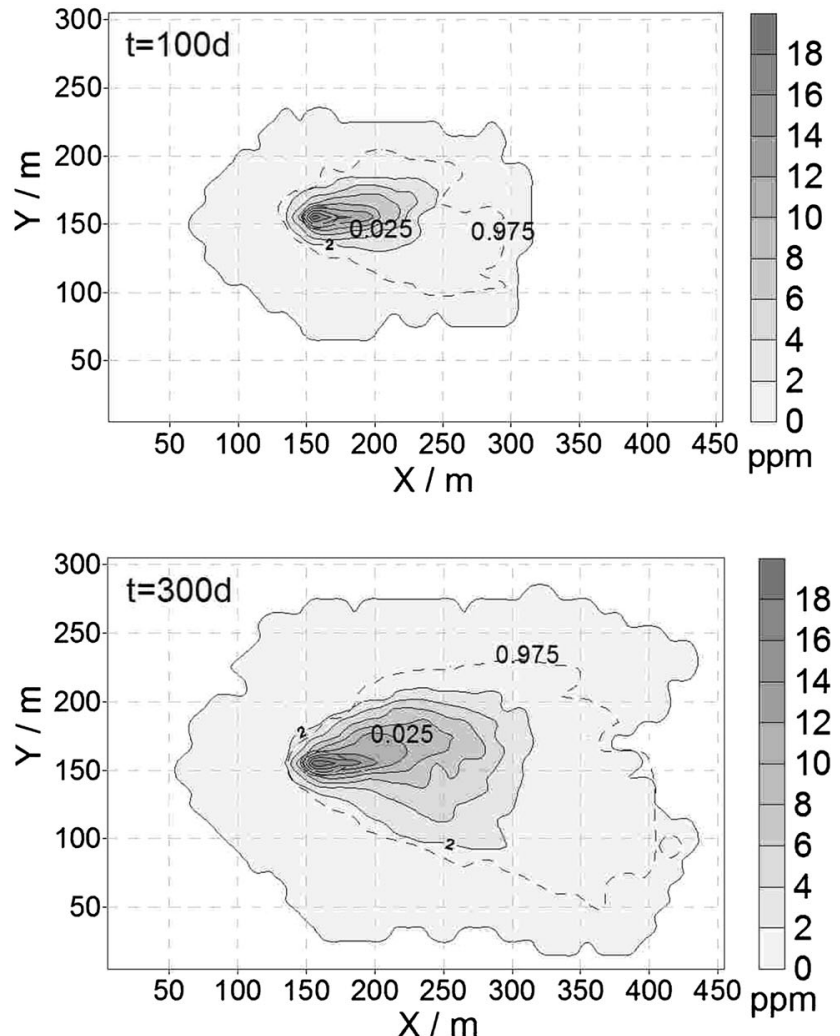

Fig. 6 Ensemble average hydraulic head and mass concentration distributions for $t=100$ days, $t=200$ days and $t=300$ days: overall predictive uncertainty 
steps, the uncertainty of predictive distribution of mass concentration $(U)$ was increased when stochastic uncertainty $\left(U_{1}\right)$ and parametric uncertainty $\left(U_{2}\right)$ was accounted for. The mass concentration contour maps also showed that there was an increase in the zone of $95 \%$ uncertainty interval along the flow direction.

As to the relative contribution of stochastic uncertainty $\left(U_{1}\right)$ and parametric uncertainty $\left(U_{2}\right)$ to the overall predictive uncertainty $(U)$ for hydraulic head and mass concentration distribution, the numerical experiment in this paper indicated that parametric uncertainty $\left(U_{2}\right)$, which was $87.7,8.99$ and $31.5 \%$ higher than stochastic uncertainty for $I_{1}, I_{2}$ and $I_{3}$, was a little more important than stochastic uncertainty $\left(U_{1}\right)$ for the predictive uncertainty of hydraulic head $(U)$. However, when the uncertainty of hydraulic head as well as macrodispersion was transported to mass transport model, a much bigger contribution of stochastic uncertainty $\left(U_{1}\right)$ than parametric uncertainty $\left(U_{2}\right)$ to the overall predictive uncertainty for mass concentration distribution $(U)$ was observed. For the simulation time of 100 days, the stochastic uncertainty was $2.43,16.24$ and $14.06 \%$ higher than parametric uncertainty for $I_{1}$, $I_{2}$ and $I_{3}$. For the simulation time of 200 days, $6.23 \%$, $7.50 \%$ and $6.65 \%$ higher of stochastic uncertainty than parametric uncertainty was observed. And for the simulation time of 300 days, 20.66, 9.13 and $4.03 \%$ higher of stochastic uncertainty than parametric uncertainty was obtained.

\section{Conclusion}

Bayesian approach is applied in the paper to quantify parameter uncertainty and its impact on hydraulic head and mass transport in heterogeneous aquifer. Specifically, we focus on inferring parameters in RSF and their effects on macrodispersion. Two types of uncertainty, parametric uncertainty and stochastic uncertainty, are addressed. The simulation results of a hypothetical two-dimensional groundwater flow and mass transport show that the inferred posterior probability distribution is updated by Bayesian approach. The posterior distributions are not obeyed to uniform distributions. As to the relative contribution of stochastic uncertainty and parametric uncertainty to the overall predictive uncertainty for hydraulic head and mass concentration distribution, the numerical experiment in this paper indicates that parametric uncertainty is a little more important than stochastic uncertainty for the predictive uncertainty of hydraulic head. When the uncertainty of hydraulic head as well as macrodispersion is transported to mass transport model, a much bigger contribution of stochastic uncertainty is observed. Therefore, parameters uncertainty should not be neglected during the process of head and mass transport simulation in heterogeneous aquifer.

Acknowledgments The study was financially supported by the National Natural Science Foundation of China (51009063, 51039001, 50978088, 50808071) and the Scientific Research Project for the Three Gorges' Environmental Protection (SX 2010-026).

\section{References}

Box GEP, Tiao GC (1992) Bayesian inference in statistical analysis. Wiley, New York

Ezzedine S, Rubin Y (1996) A geostatistical approach to the conditional estimation of spatially distributed solute concentration and notes on the use of tracer data in the inverse problem. Water Resour Res 32(4):853-861

Feyen L Jr, Ribeiro PJ, De Smedt F, Diggle PJ (2003) Stochastic delineation of capture zones: classical versus Bayesian approach. J Hydrol 281(4):313-324

Franssen HJH, Gómez-Hernández JJ, Sahuquillo A (2003) Coupled inverse modeling of groundwater flow and mass transport and the worth of concentration data. J Hydrol 281(4):281-295

Fu JL, Gómez-Hernández JJ (2009) Uncertainty assessment and data worth in groundwater flow and mass transport modeling using a blocking Markov chain Monte Carlo method. J Hydrol 364(3-4):328-341

Gelman A, Rubin DB (1992) Inference from iterative simulation using multiple sequences. Stat Sci 7(4):457-511

Hassan AE, Bekhit HM, Chapman JB (2009) Using Markov Chain Monte Carlo to quantify parameter uncertainty and its effect on predictions of a groundwater flow model. Environ Model Softw 24(6):749-763

Kerrou J, Renard P, Hendricks HJ, Franssen H, Lunati I (2008) Issues in characterizing heterogeneity and connectivity in non-multigaussian media. Adv Water Resour 31(1):147-159

Liang J, Zeng GM, Guo SL, Li JB, Wei AL, Shi L, Li XD (2009) Uncertainty analysis of stochastic solute transport in a heterogeneous aquifer. Environ Eng Sci 26(2):359-368

Liang J, Zeng GM, Guo SL, Wei AL, Li XD, Shi L, Du CY (2010) Optimal solute transport in heterogeneous aquifer: coupled inverse modelling. Int J Environ Pollut 42(1-3):258-269

Rojas R, Kahunde S, Peeters L, Batelaan O, Feyen L, Dassargues A (2010) Application of a multimodel approach to account for conceptual model and scenario uncertainties in groundwater modeling. J Hydrol 394(3-4):416-435

Sudicky EA (1986) A natural gradient experiment on solute transport in a sand aquifer: spatial variability of hydraulic conductivity and its role in the dispersion process. Water Resour Res 22(13):2069-2082

Sun NZ (1994) Inverse problems in groundwater modeling. Kluwer Academic Publishers Group, Norwell

Wilson JL, Miller PJ (1978) Two-dimensional plume in uniform ground water flow. J Hydraul Div 104(4):503-514 
Yeh WWG (1986) Review of parameter identification procedures in groundwater hydrology: the inverse problem. Water Resour Res 22(1):95-106

Yeh WWG, Yoon YS (1981) Aquifer parameter identifiability with optimum dimension in parameterization. Water Resour Res 17(3):664-672
Zeng GM, Liang J, Guo SL, Shi L, Xiang L, Li XD, Du CY (2009) Spatial analysis of human health risk associated with ingesting manganese in Huangxing Town, Middle China. Chemosphere 77(3-4):368-375 